MEI

II, vol. 6 n⿳⺈ 10

\title{
Panorámica general del uso de Sistemas Integrados de Gestión Bibliotecaria en España
}

\author{
Sara Picazo Cháfer
}

\section{Resumen}

El propósito del estudio ha sido conocer los Sistemas Integrados de Gestión Bibliotecaria (SIGB) utilizados en España. Para ello se han agrupado las

Recibido el 12-06-2015

Aceptado en 06-07-2015 bibliotecas según su tipología, para poder analizar si el uso de estos sistemas tiene relación con las necesidades de las mismas. La evolución tecnológica de las últimas décadas, ha favorecido el nacimiento de nuevos formatos basados en los medios digitales y el desarrollo de nuevos sistemas de este tipo basados en el software libre. Por lo tanto, también se pretende reflejar si durante los últimos años ha aumentado el uso de sistemas libres frente al uso habitual de sistemas de carácter propietario.

\section{Palabras clave}

Sistemas Integrados de Gestión Bibliotecaria, SIGB, software libre, software propietario, automatización de bibliotecas.

\section{Title}

General overview of use of Integrated Systems Library Management in Spain .

\begin{abstract}
The purpose of the study has been to learn the integrated library systems (ILS) used in Spain. In order to do so, the libraries have been grouped by their typology, to analyze if the use of these systems is related to the needs of the same. Technological developments of the last decades have favored the emergence of new formats based on digital media and the development of new systems of this type based on free software. Therefore, it is also intended to reflect if in recent years has increased the use of open systems versus standard systems of proprietary nature.
\end{abstract}

\section{Keywords}

Integrated Library Systems, ILS, Open Software, Proprietary Software, Library automation 


\section{1.- ANTECEDENTES}

Los Sistemas Integrados de Gestión Bibliotecaria (a partir de ahora SIGB) tienen su origen en Norteamérica alrededor de los años 60, pero no se empiezan a comercializar hasta principios de la década de los 80. Estamos hablando de casi 35 años de evolución tecnológica que ha ido paralela al desarrollo de estos sistemas. En España su desarrollo es posterior al de otros países occidentales, debido al vacío cultural que produjo la dictadura, de manera que nuestros inicios en este campo se sitúan a raíz del Estado Democrático (Galán, 2012).

Los cambios que han sufrido estos sistemas responden a la evolución tecnológica del momento. Según Játiva (2002), Arriola y Butrón (2008) y Gutiérrez (2001) se distinguen cuatro etapas, cada una de ellas caracterizada por la aparición de un avance trascendental como el formato MARC, los circuitos integrados, el CD-ROM y la consolidación de Internet como medio de comunicación universal.

Desde la última generación hasta la actualidad ha transcurrido más de una década, en la que el desarrollo tecnológico en el campo de la informática ha favorecido la aparición de nuevos formatos, nuevos tipos de licencias, sistemas de almacenamiento diferentes, etc. Tal y como señalan Valentín Ruiz, Fernández Sánchez y Torre-Martín (2012) cloud computing (informática en la nube) es un servicio que permite ejecutar desde Internet distintas aplicaciones sin tenerlas instaladas en el servidor local. Este nuevo reto surge motivado por el aumento de contenidos digitales, la aparición del libro electrónico y la proliferación de las bibliotecas virtuales o digitales. El problema reside en que los sistemas actuales no están completamente capacitados para la circulación de contenidos electrónicos, aunque en estos momentos las empresas están desarrollando nuevas prestaciones y actualizando sus versiones para que se adapten a las necesidades actuales de los usuarios.

El mercado de estos programas ha estado dominado siempre por los sistemas de carácter propietario, pero a partir del 2006 los sistemas libres hacen su aparición en el mercado estadounidense, extendiéndose de forma paulatina por todo el mundo. En la actualidad, los SIGB libres están experimentando un desarrollo más técnico y en consecuencia su aplicación está teniendo un considerable aumento, siendo Koha el sistema más desarrollado e implantado a nivel mundial. 


\section{2.-METODOLOGÍA}

La obtención de los datos se ha llevado a cabo en tres fases. En la primera, se ha procedido a buscar y seleccionar los centros de documentación y bibliotecas que forman el corpus del estudio. Se accedió al directorio de bibliotecas facilitado por el Ministerio de Educación, Cultura y Deporte (www.mcu.es). Estas agrupaciones se ampliaron con los listados facilitados por el directorio de la Biblioteca Nacional de España (www.bne.es). Además, para incluir algunas tipologías no recogidas en los directorios anteriores, como las bibliotecas de los Ministerios y las bibliotecas gubernamentales, se consultaron las páginas web de las diputaciones o del gobierno central de las provincias españolas.

En la segunda fase, se procedió a recoger el dato que se requería, que era saber el SIGB que utilizaban para gestionar su centro. En segundo lugar, se accedió a las webs de las empresas desarrolladoras de los sistemas, para complementar los datos con información de la propia compañía sobre sus clientes y la distribución del software.

La tercera fase consistió en establecer una clasificación propia de los SIGB, ya que a través de las páginas web sus empresas desarrolladoras se observó que debido a los movimientos tan frecuentes dentro de este mercado, podían haber confusiones con las fusiones y absorciones que se han producido en los últimos 10 años. En muchas ocasiones, las bibliotecas no tienen conocimiento de ello y no saben exactamente qué sistema están utilizando ni la versión del mismo (que a veces cambia completamente de nombre). Debido a estos matices se van a clasificar los SIGB que han sufrido modificaciones de este tipo bajo el siguiente criterio:

- Innovative Interfaces: el producto estrella de esta empresa es Millennium, pero ha surgido una plataforma nueva llamada Sierra que sustituye la versión anterior. Algunos clientes ya han adquirido el nuevo sistema, pero la mayoría sigue considerando Millennium como su sistema de gestión. Todos los centros que utilicen uno de estos dos sistemas se engloban bajo Millennium.

Baratz Servicio de Telecomunicaciones, S.A.: esta empresa desarrolla diferentes sistemas, ya sean de gestión bibliotecaria, de gestión documental, de gestión de archivos, etc. En el estudio vamos a diferenciar entre Absys, AbsysNet y Absys Express. 
OdiloTID y 3000 informática: son dos empresas diferentes pero ambas con sede en Cartagena. 3000 informática ha sido una empresa fuerte dentro de este sector en España, pero actualmente se centra más en la formación profesional. OdiloTID, de nacimiento más reciente, se centra en la distribución y gestión de los programas que desarrolla y en dar soporte a los centros que utilizaban Biblio 3000 (producto de 3000 Informática). Son productos diferentes, pero en algunos OPACs figura el nombre de ambas empresas, ya que los centros que utilizaban Biblio 3000 tienden a cambiar de software, siendo OdiloTT el más escogido por las facilidades que se dan para la migración. No obstante, algunos centros siguen trabajando con el modelo antiguo comercializado por 3000 Informática, que serán respetados y clasificados bajo Biblio 3000. El resto de centros que hayan actualizado el programa y figuren ambas empresas en su OPAC se engloban bajo OdiloTT.

Amicus-Librisuite y Librisuite: la fusión entre Scanbit y Sabini dio lugar a la compañía Libritech Ibérica. Las empresas siguieron respetando los sistemas comercializados añadiendo el término Librisuite (Sabini-Librisuite y Amicus-Librisuite). En la actualidad, Sabini-Librisuite es un programa algo obsoleto pero que se sigue utilizando, mientras que Amicus-Librisuite y Librisuite se engloban bajo Librisuite.

Techlib y Livelink: Open Text es la desarrolladora del programa Livelink ECM Library Management, que anteriormente era conocido como Techlib. Estos programas se pueden utilizar por separado o fusionando sus módulos. La mayoría de bibliotecas españolas siguen utilizando Techlib, por lo tanto se respetan ambos programas.

- Inmagic DB/TextWorks e InmagicTOT: el primer sistema es un gestor documental que incluye módulos que facilitan las tareas bibliotecarias. A partir de este sistema se desarrolló InmagicTOT que sí que es un SIGB propiamente dicho. Esta es la razón por la que muchas bibliotecas no saben exactamente qué sistema de los dos utilizan, por lo tanto se engloban todos los centros bajo Inmagic.

- Koha y Koha-Kobli: este último es una versión de Koha que se ha desarrollado en España bajo las necesidades de las bibliotecas españolas, por lo tanto se engloban todas bajo Koha. 
Propio: algunos centros, simplemente, han informado de que disponen de un desarrollo propio y se engloban como tal.

\section{3.-RESULTADOS}

\begin{tabular}{|l|c|c|c|c|}
\hline & Consultas & Respuestas & Utilizan & No utilizan \\
\hline Bibliotecas de carácter General y Público & & & & \\
\hline Catálogos Colectivos y Redes Bibliotecarias & 61 & $58(95 \%)$ & $58(100 \%)$ & 0 \\
\hline B. Nacional y Autonómicas & 15 & $15(100 \%)$ & $15(100 \%)$ & 0 \\
\hline B. Públicas del Estado & 53 & $53(100 \%)$ & $53(100 \%)$ & 0 \\
\hline B. Virtuales y Digitales & 45 & $40(89 \%)$ & $37(92.5 \%)$ & 3 \\
\hline Bibliotecas de Enseñanza & & & & \\
\hline B. Académicas & 180 & $167(93 \%)$ & $148(89 \%)$ & 19 \\
\hline B. de Instituciones de Enseñanza Superior & 73 & $54(74 \%)$ & $48(88 \%)$ & 6 \\
\hline B. Universitarias & 80 & $78(97.5 \%)$ & $76(97 \%)$ & 2 \\
\hline B. Escolares & 350 & $239(68 \%)$ & $221(92 \%)$ & 18 \\
\hline Bibliotecas del Gobierno & & & & \\
\hline B. de los Ministerios & 36 & $33(92 \%)$ & $33(100 \%)$ & 0 \\
\hline B. Gubernamentales & 120 & $96(80 \%)$ & $91(95 \%)$ & 5 \\
\hline Bibliotecas Especializadas & & & & \\
\hline Centros de Documentación y B. de Investigación & 150 & $135(90 \%)$ & $103(76 \%)$ & 32 \\
\hline B. de Museos & 782 & $545(70 \%)$ & $242(44 \%)$ & 303 \\
\hline B. de Instituciones Religiosas & 67 & $37(55 \%)$ & $28(75 \%)$ & 9 \\
\hline B. de Centros Sanitarios & 95 & $70(74 \%)$ & $47(67 \%)$ & 23 \\
\hline B. de Empresas y Firmas Comerciales & 50 & $17(34 \%)$ & $11(64 \%)$ & 6 \\
\hline \multicolumn{1}{|c|}{ TOTALES } & $2.157(24 \%)$ & $1.637(76 \%)$ & $1.211(74 \%)$ & $426(26 \%)$ \\
\hline
\end{tabular}

\section{1.-Bibliotecas de carácter general y público}

\subsection{1.- Catálogos colectivos y redes bibliotecarias}

Los catálogos colectivos y las redes bibliotecarias se diferencian de las demás agrupaciones analizadas porque se componen de diferentes centros, que comparten un catálogo bibliográfico automatizado bajo un sistema de estas características. La gestión colectiva de bibliotecas requiere prestaciones diferentes y sistemas más potentes. No obstante, las bibliotecas que forman parte de estos colectivos en su propio centro pueden utilizar otro sistema, aunque esto supone un esfuerzo extra, ya que los fondos deben ser exportados a ambos sistemas (al del catálogo colectivo y al propio individual). 


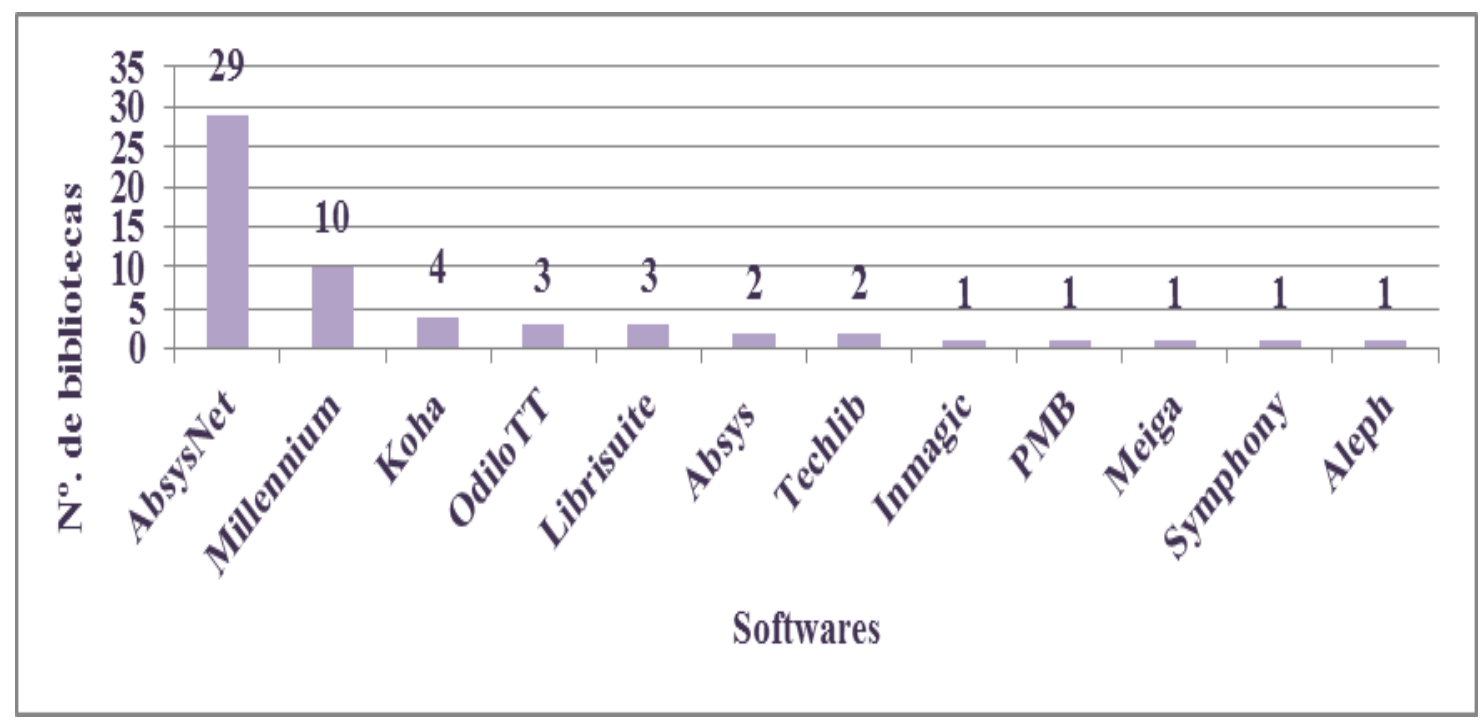

\subsection{2.- Biblioteca Nacional y autonómicas}

AbsysNet es el sistema más utilizado (80\%) a excepción de Cataluña que se decanta por el SIGB de Innovative Interfaces (Millennium). La Biblioteca Nacional de España también opta por un sistema diferente al de las centrales: Symphony, un sistema americano utilizado principalmente en centros de carácter universitario.

\subsection{3.- Bibliotecas Públicas del Estado}

Dentro de esta agrupación utilizan todas AbsysNet (90\%) a excepción de Cataluña (que utilizan en las 3 Millennium) y Ceuta y Mallorca que trabajan con Absys.

\subsection{4.- Bibliotecas virtuales $y$ vigitales}

Esta tipología utiliza principalmente Digibib, tan sólo 3 centros no utilizan SIGB. Las bibliotecas que no utilizan dichos sistemas emplean gestores de objetos digitales como Dspace, ya que estos gestores se desarrollaron antes de que los SIGB se empezaran a adaptar a este cambio.

\section{2.- Bibliotecas de enseñanza}

\subsection{1.- Bibliotecas académicas}

Dentro de esta rama encontramos una gran variedad de programas, algunos de ellos se utilizan en casos aislados, mientras que determinados grupos de academias o escuelas se decantan por un sistema en concreto. Se han incluido las Escuelas Oficiales de Idiomas de todas las provincias (en algunos casos más de una EOI por provincia) y las Escuelas de Arte, estos centros al ser de 
carácter público se ajustan a los programas facilitados por las Consejerías de Educación a los centros escolares. Por esta razón Abies tiene bastante peso dentro de esta tipología (24\%).Los Ilustres Colegios de Abogados, Arquitectos, etc., se han decantado principalmente por el sistema Biblio 3000 (6.5\%). Sin embargo, como este sistema se está quedando obsoleto la mayoría de centros tienden a cambiar a OdiloTT $(15 \%)$.

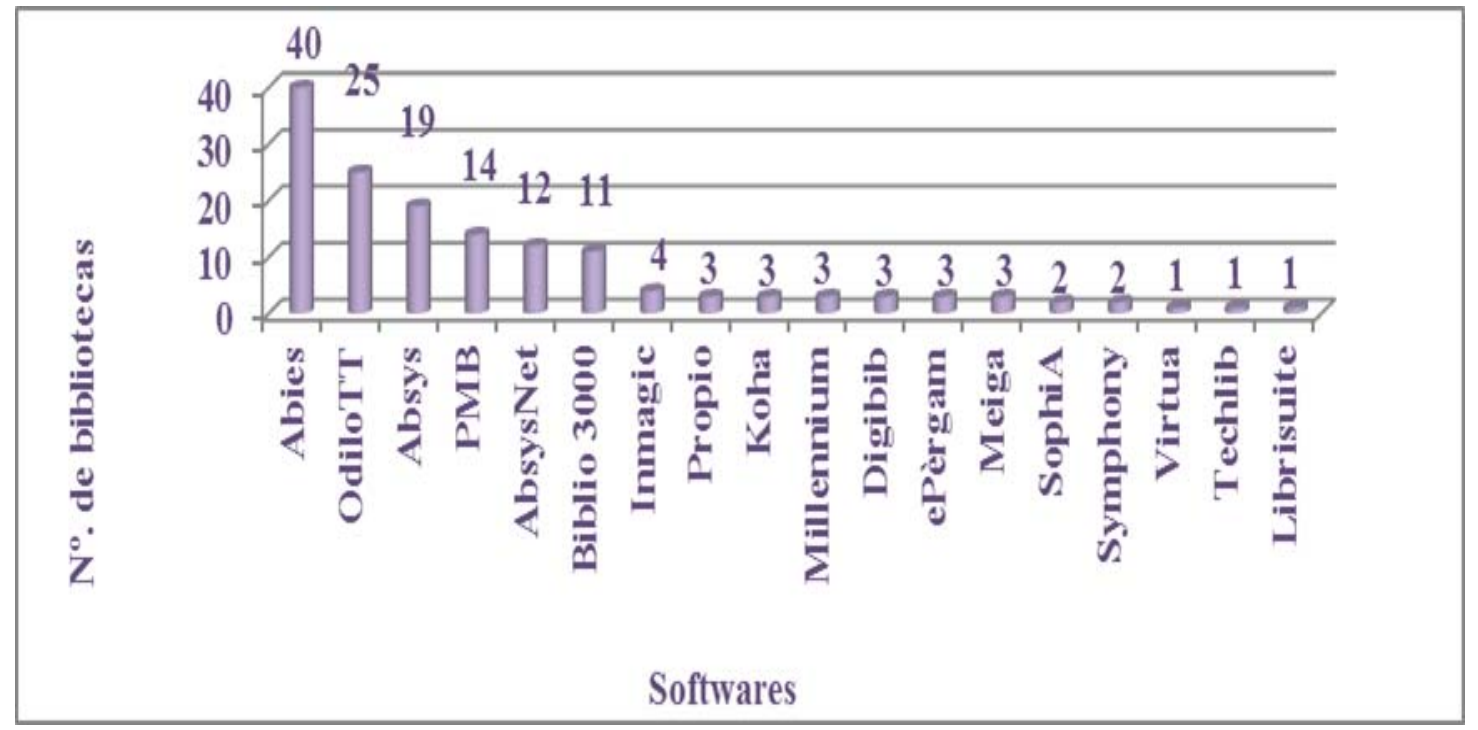

\subsection{2.- Bibliotecas de instituciones de enseñanza superior}

La empresa Baratz Telecomunicaciones, S.A. también aglutina en esta tipología la automatización de la mayoría de centros con sus dos productos estrella: Absys (22\%) y AbsysNet (15\%). Millennium (11\%) y Symphony (2\%) son sistemas con prestaciones orientadas a centros de carácter universitario, por eso su presencia en esta tipología es más acentuada que en otras agrupaciones.

\subsection{3.- Bibliotecas universitarias}

Las prestaciones de las bibliotecas universitarias son las más potentes en comparación con las necesidades de las demás tipologías bibliotecarias. Se observa una clara decantación por el sistema de Innovative Interfaces (algunos centros han adquirido ya su última actualización Sierra), mientras que la mayoría sigue utilizando Millennium.

AbsysNet es el sistema utilizado para REBIUN (Red de Bibliotecas Universitarias Españolas), por esta razón la presencia de AbsysNet (14\%) sigue siendo notable en esta tipología. No obstante, las redes bibliotecarias universitarias registradas por comunidades utilizan Millennium.Symphony es 
un sistema potente que no es muy utilizado a nivel general en otras tipologías, pero en la Comunidad de Madrid se celebró una reunión entre los representantes de la mayoría de universidades de la comunidad, donde se escogió este sistema.

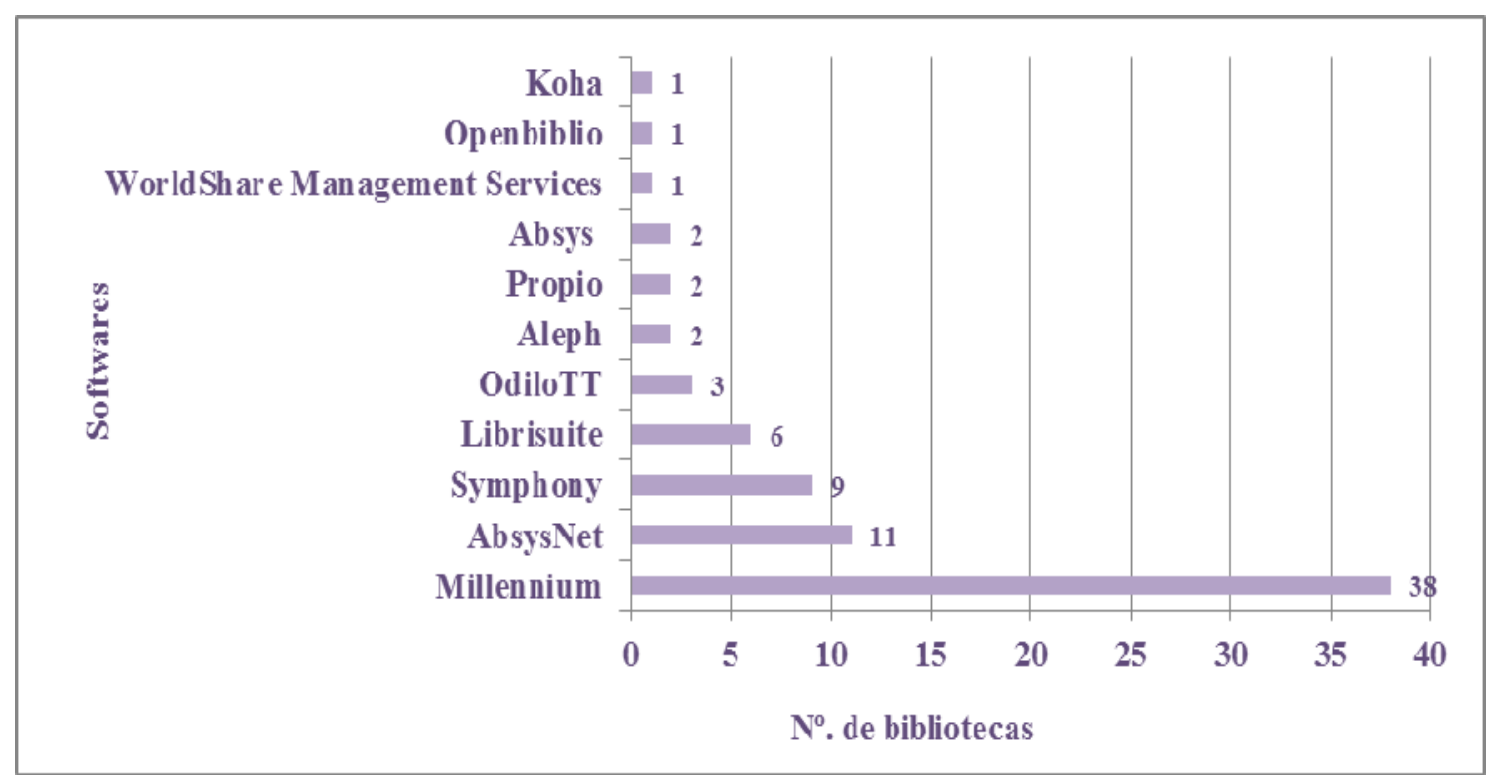

\subsection{4.-Bibliotecas escolares}

Los centros escolares comprenden un grupo amplio, aunque la biblioteca no está desarrollada al nivel que en los grupos anteriores. A pesar de ser numerosos centros con necesidades comunes, son escasas las redes bibliotecarias con catálogos colectivos. Existen cuatro redes bibliotecarias que utilizan SIGB, dos de ellas utilizan Koha, una Absys.edu y otra AbsysNet. Dentro de esta tipología todas las bibliotecas utilizan sistemas gratuitos o libres proporcionados por las Consejerías de Educación: Abies (a nivel nacional), Meiga (Galicia), PMB (Comunidad Valenciana) y ePèrgam (Cataluña).

\section{3.- Bibliotecas del Gobierno}

\subsection{1.- Bibliotecas de los ministerios}

Para ser un grupo tan reducido existe bastante variedad de sistemas, aunque Absys sigue siendo el sistema más utilizado con un 36\% seguido de Koha con un $27 \%$. Existen dos desarrollos propios y dos casos aislados de sistemas un poco obsoletos como Sabini-Librisuite y Liber-Marc. Finalmente, Millennium también es utilizado en esta tipología, aunque su presencia es más bien baja 


\subsection{2.- Bibliotecas gubernamentales}

Absys (25\%) y AbsysNet (12.5\%) son los sistemas más utilizados junto OdiloTT (25\%). Los demás sistemas se utilizan aisladamente, siendo Techlib (9\%), Millennium (7\%) y Koha (5\%) los utilizados en una proporción mayor. Dentro de esta tipología encontramos una red de bibliotecas muy importante como es el CENDOJ (Red de bibliotecas del Poder Judicial). Todas ellas utilizan el sistema AbsysNet y, en algunos casos Absys. Por lo tanto, la presencia de estos sistemas sería más si se tiene en cuenta este dato.

\section{4.- Bibliotecas especializadas}

\subsection{1.- Centros de documentación y bibliotecas de investigación}

La mayoría de estos centros forman parte de redes bibliotecarias, por eso prima el uso de Absys (19\%) y AbsysNet (14\%) a nivel general y de Millennium (9\%) en Cataluña. No obstante, hay una gran variedad de sistemas. Dentro de las bibliotecas de investigación se debe tener en cuenta la presencia de una de las redes bibliotecarias más importantes de España como son las bibliotecas del Consejo Superior de Investigaciones Científicas (CSIC). En los directorios que se han consultado para extraer los centros sí que figuraban algunas bibliotecas pertenecientes a esta red, pero el consejo comunicó que todas sus bibliotecas estaban automatizadas bajo el mismo sistema (Aleph). Alrededor de 100 bibliotecas especializadas trabajan bajo el SIGB Aleph, que es uno de los sistemas más antiguos que existen en este sector.

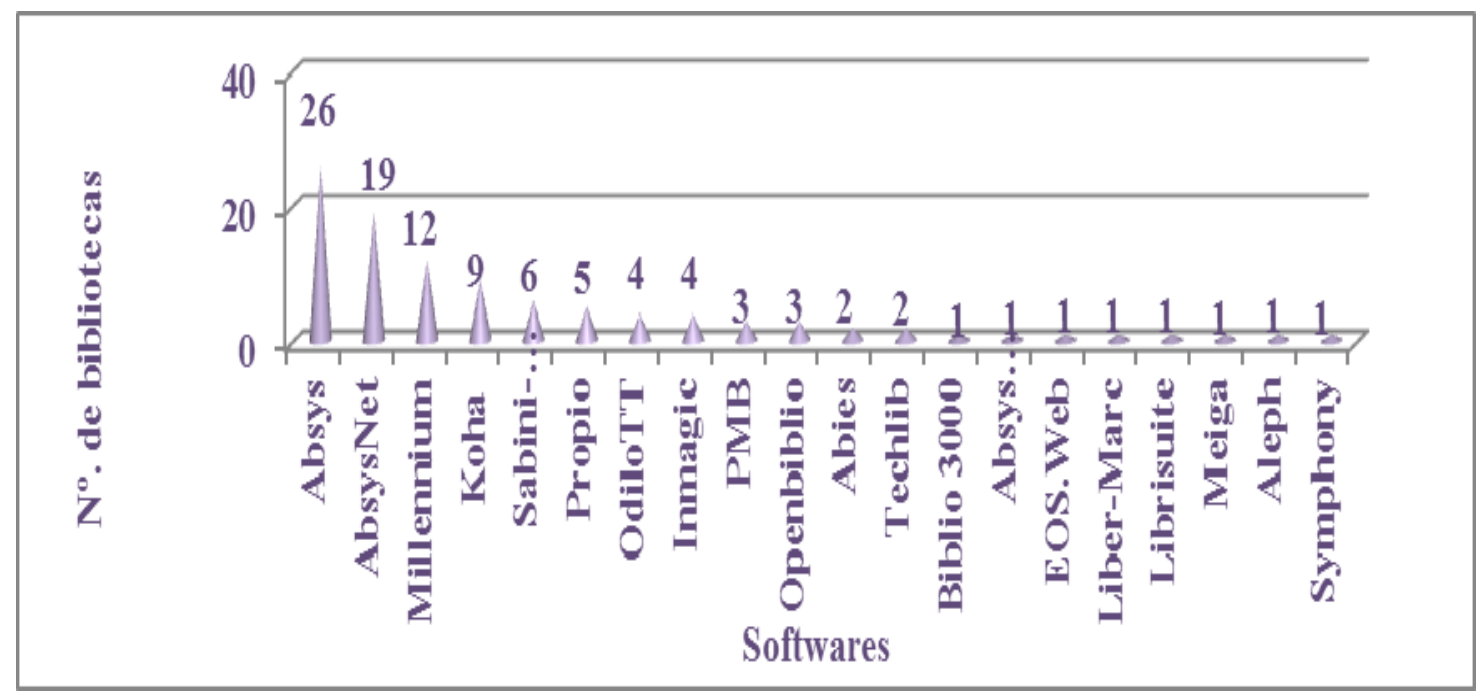




\subsection{2.- Bibliotecas de museos}

A partir del gráfico podemos observar que existe una gran variedad de softwares, pero la realidad es que la tabla inicial muestra claramente que esta tipología tiene un considerable número de museos que no utilizan SIGB: 242 $(55 \%)$. Se debe tener en cuenta que una gran parte de museos no dispone de fondos bibliográficos, ya que muchos de los incluidos eran parques naturales, casas rurales, etc. Aquellos centros cuya tipología fundamental son los objetos, utilizan otros sistemas de gestión adaptados a sus necesidades como puede ser Domus o MuseumPlus. Los sistemas más utilizados dentro del grupo de museos que sí utiliza SIGB es Absys (12\%) y AbsysNet (16\%). Esta elección también responde a un motivo, dado que la mayoría de museos pertenece a redes bibliotecarias, bien sea BIMUS que es la más importante dentro de esta tipología o a las redes públicas de cada comunidad que se decantan también por estos sistemas.

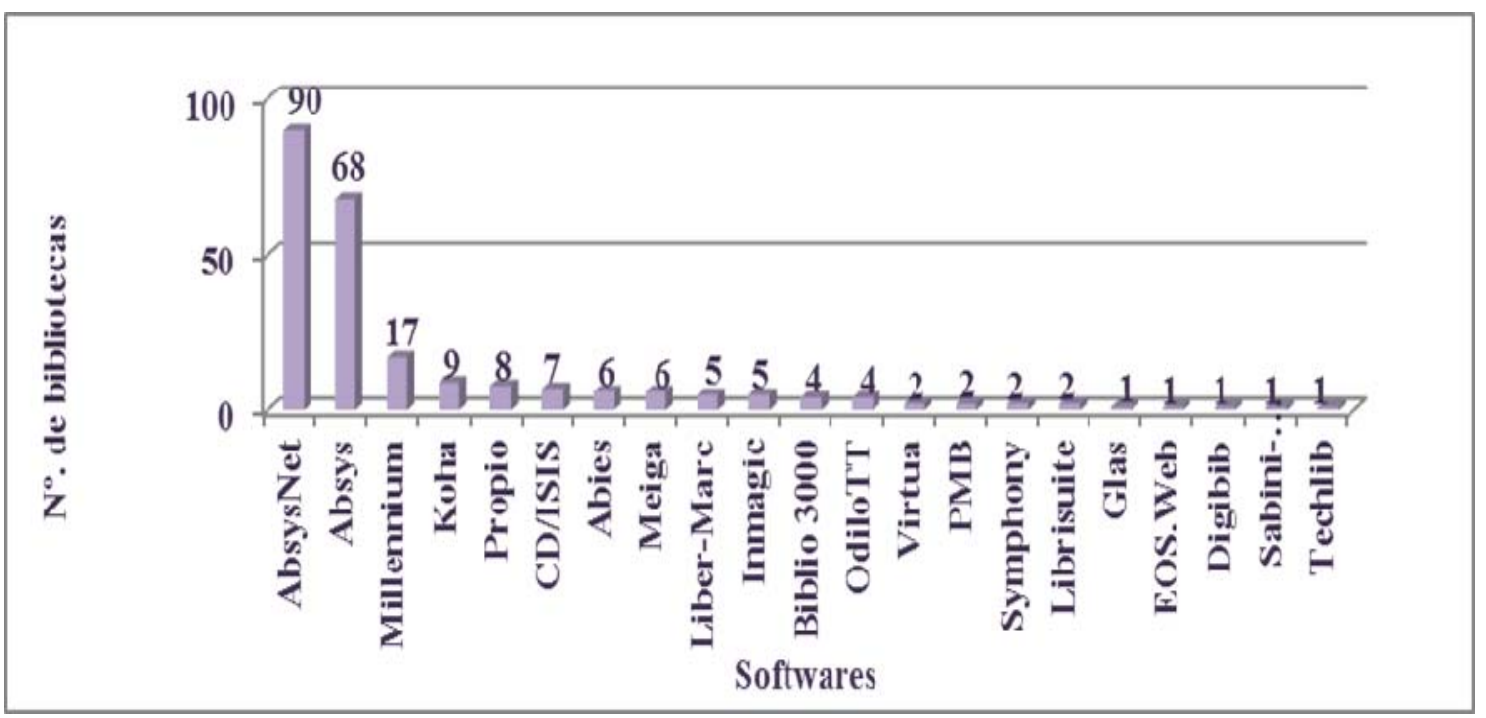

\subsection{3.- Bibliotecas de instituciones religiosas}

La mayoría de estos centros no disponen de fondos económicos para hacer frente a un sistema potente que gestione sus fondos bibliográficos, por eso de las bibliotecas consultadas un $24 \%$ no están automatizadas con SIGB. El sistema más utilizado es Millennium (16\%) seguido de Absys (13\%) y Koha $(11 \%)$.

\subsection{4.- Bibliotecas de centros sanitarios}

Las necesidades de estos centros son muy diferentes a las demás agrupaciones, la tipología documental más importante son las publicaciones seriadas, en su mayoría las revistas electrónicas. Dentro de esta rama se observa que el 
sistema más utilizado es EOS.Web (27\%) (Sucesor de Glas). El centro que utiliza la versión anterior de EOS.Web, explica que la ventaja de este sistema reside en que se puede comprar por módulos. El programa actual no sabemos si sigue prestando esta facilidad, pero a partir de la web de Sibadoc que es el distribuidor de EOS.Web en España, informan que el módulo de las publicaciones periódicas es la principal herramienta del sistema, que hace que el control de éstas sea sencillo. Existen dentro de esta tipología dos redes bibliotecarias: el Servicio Gallego de Salud que utiliza EOS.Web y AsturSalud que utiliza AbsysNet. Este último sistema es utilizado en otros hospitales, por lo que su presencia en esta agrupación también es bastante visible (21\%).

\subsection{5.- Bibliotecas de empresas y firmas comerciales}

La respuesta por parte de esta tipología ha sido muy reducida, tan sólo 17 centros $(34 \%)$ de los 50 consultados han facilitado el dato requerido. Se ha incluido esta pequeña agrupación para que hubiese conocimiento de este tipo de centros y que sus necesidades bibliográficas también les conducen a utilizar sistemas de este tipo para gestionar su información.

\section{5.- Uso de Sistemas Integrados de Gestión Bibliotecaria libres frente propietarios}

En España el mercado sigue estando dominado por los SIGB de carácter propietario. Los sistemas de carácter libre tienen una corta trayectoria, en la que poco a poco han ido incorporando los estándares y protocolos generales. Es importante diferenciar entre SIGB de carácter libre y SIGB simplemente gratuitos. Éstos últimos son sistemas desarrollados por las consejerías de educación u organizaciones, que realizan este trabajo de forma voluntaria para facilitar un programa de estas características a bibliotecas con poco recursos económicos, como se ha podido observar en las bibliotecas escolares. Estos sistemas son gratuitos pero no se pueden modificar y, en la mayoría de los casos, se deben realizar cursos de formación para su adquisición. Dentro de este grupo encontramos varios sistemas utilizados en nuestro país: Abies, Meiga, ePèrgam y CD/ISIS.

Los SIGB libres son aquellos que permiten modificar su código fuente, además de dar independencia al cliente en cuanto al mantenimiento del sistema, es decir, el soporte técnico puede ser realizado por la empresa desarrolladora del software o por cualquier otra y, también son gratuitos. En España se utilizan 3 sistemas de estas características: Koha, PMB y Openbiblio. Como ya se ha comentado anteriormente, en nuestro país se ha desarrollado una versión nueva de Koha denominada Kobli. Este sistema es el más utilizado en nuestro marco geográfico (dentro del grupo de softwares libres). De hecho en los 
últimos años han ido incorporándose a este nuevo sistema bastantes bibliotecas, de diferentes tipologías e incluso es utilizado por redes bibliotecarias y bibliotecas universitarias, lo que manifiesta los avances de este sistema en los últimos años. Los otros dos sistemas se utilizan en casos más aislados, aunque PMB en la Comunitat Valenciana y Cataluña se utiliza en todos los centros con características similares a las bibliotecas escolares. En definitiva, aunque más desarrollados y en continua evolución, dichos sistemas están todavía muy por detrás de los sistemas de carácter propietario en términos de instalaciones como podemos observar en la siguiente tabla.

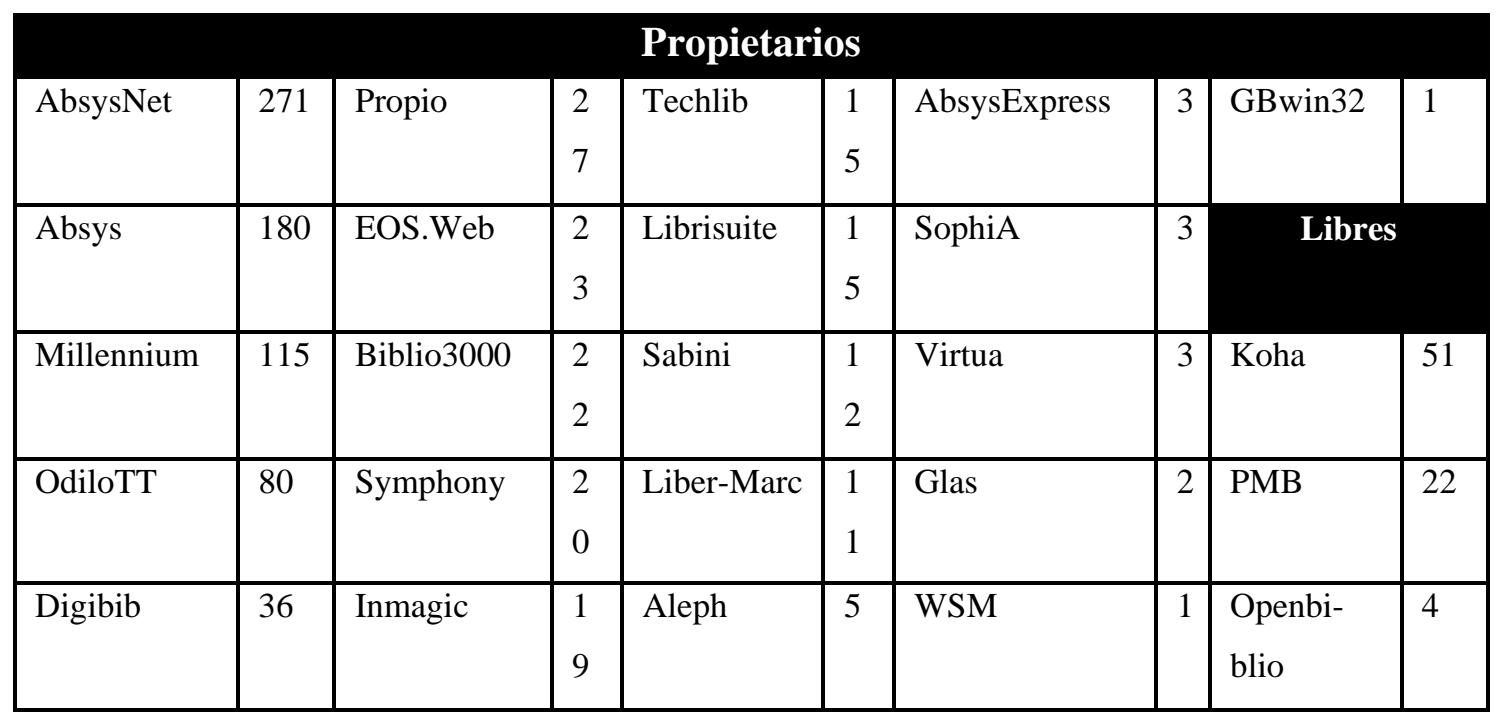

\section{4.- COCLUSIONES}

Dentro del marco geográfico español coexisten una gran variedad de sistemas, donde la empresa Baratz Telecomunicaciones S.A. es la que aglutina el mayor número de centros bajo sus dos productos estrella Absys y AbsysNet. El lanzamiento de éste último en 2004 revolucionó el panorama español, ya que fue el primer sistema nacional que permitía la gestión de un gran grupo de bibliotecas de forma conjunta. Trabajar en red bajo un mismo sistema aporta beneficios tanto económicos como profesionales, por este motivo muchas bibliotecas con recursos limitados cuando las circunstancias les permiten automatizarse o adquirir un sistema más desarrollado, deciden incorporarse a redes bibliotecarias para nutrirse de los beneficios del trabajo en grupo y que el desembolso económico sea más asequible. 
La empresa Innovative Interfaces con su producto Millennium también tiene una destacada presencia en nuestro país, sobre todo en la comunidad catalana, donde acapara un elevado número de instalaciones respecto a los demás sistemas. Este hecho pone de manifiesto que otro nexo de conexión en la elección de un SIGB, son las Comunidades Autónomas y los sistemas utilizados en esos marcos territoriales. Ejemplos de ello son la comunidad catalana con Millennium, PMB y ePèrgam; la Comunitat Valenciana con Techlib y PMB; Galicia con Meiga; Extremadura con OdiloTT y el País Vasco con AmicusLibrisuite. En ocasiones, esta norma no se corresponde con el sistema que mejor gestionaría las necesidades de la biblioteca, pero cuando una comunidad se decanta por un SIGB es, principalmente, porque ha llegado a un acuerdo con la compañía distribuidora para la compra de un número de licencias. En otros casos las Consejerías de Educación desarrollan sistemas propios para distribuirlos gratuitamente entre las bibliotecas de su comunidad que tienen pocos recursos económicos.

Las tipologías bibliotecarias también tienen una importante asociación con el tipo de SIGB que se utiliza para la gestión de determinadas bibliotecas con características comunes. Las bibliotecas universitarias son las que utilizan los SIGB más potentes del mercado actual: Millennium, AbsysNet y Symphony. En otras tipologías también se observa este fenómeno: las bibliotecas de centros sanitarios se decantan por el sistema EOS.Web; las redes bibliotecarias y catálogos colectivos por AbsysNet; las bibliotecas virtuales por Digibib; las bibliotecas escolares por Abies. Así pues, podemos afirmar que existen tres circunstancias que influyen en la selección de un sistema: la incorporación a una red bibliotecaria o catálogo colectivo, la comunidad autónoma a la que se pertenece y la tipología bibliotecaria.

Finalmente, destacar que España sigue siendo un país dominado por los SIGB de carácter propietario, aunque los sistemas de carácter libre van aumentando sus instalaciones en todas las tipologías bibliotecarias, siendo Koha en su versión Kobli la más utilizada en nuestro país. Este avance hacia el software libre parece ser desconocido por un gran número de bibliotecas, ya que los datos muestran que un 26\% de los centros consultados no utilizan sistemas de estas características para gestionar sus fondos bibliográficos. Los motivos son: la falta de recursos económicos y la escasez de fondos bibliográficos (en el segundo caso advierten que la adquisición de este tipo de programas supone un desembolso económico grande para gestionar un número reducido de materiales), pero con los SIGB de carácter libre podrían solventar estas barreras sin ningún problema. 


\section{5.-BIBLIOGRAFÍA}

ARRIOLA, O. y BUTRON, K., 2008. Sistemas integrales para la automatización de bibliotecas basados en software libre. ACIMEDnoviembre, 18(6). [en línea] [Consulta: 14 de febrero de 2014]. Disponible en: http://bvs.sld.cu/revistas/aci/vol18_6_08/ acio91208.htm

GALÁN, E. M., 2012. Automatización Bibliotecaria en España.[en línea] [Consulta: 21 de febrero de 2014]. Disponible en:http://www.alquiblaweb.com/2012/11/08/ automatizacion-bibliotecaria-en-espana/\#

GUTIERREZ CORAL, L. A., 2001. Estudio comparativo de los Sistemas Integrados de código abierto para biblioteca: Koha y Phpmybibli.. Perú:Universidad Nacional Mayor de San Marcos.

JÁTIVA MIRALLES, M. V., 2002. Opac-portal: una nueva forma de ofrecer los recursos y servicios de la biblioteca.El profesional de la información (6)1 1: 442-453.

VALENTÍN RUIZ, F.J., FERNÁNDEZ SÁNCHEZ, M.J., y TORRE-MARÍN, R., 2012. Servicios cloud computing para la gestión de bibliotecas : hacia un nuevo modelo. [en línea]. En: $6^{\circ}$ Congreso Nacional de Bibliotecas Públicas. Burgos: Subdirección General de Coordinación Bibliotecaria.[consulta: 13 febrero de 2014] Disponible en: http:// travesia.mcu.es/portalnb/jspui/bitstream/10421/6743/1/cloud_computing.pdf 